\title{
The Impact of Maternal, Perinatal, and Early Infancy Period on the Eruption Timing of the First Primary Tooth
}

\author{
Aikaterini I. Georgiadou, Athanasios Ritsas, and Aristidis Arhakis
}

\section{ABSTRACT}

\begin{abstract}
Aim: The aim of this study is to identify and review the literature on effect of perinatal and early infancy factors on the eruption chronology of the first deciduous tooth.
\end{abstract}

\begin{abstract}
Materials and methods: A PubMed database literature survey regarding the influence of maternal, perinatal and early infancy period factors on eruption timing of the first primary tooth covering the period from January 1964 to 2020 was conducted.
\end{abstract}

Results: Maternal factors such as smoke exposure during pregnancy, gestational age or even Vitamin D levels could possibly affect the eruption timing. Strong association was evident between some perinatal factors like infant's birth weight or ethnicity and primary teeth eruption chronology, as opposed to infant's birth length or sex. Many studies indicated that prematurity could lead to delayed eruption timing, while other studies do not confirm that allegation. Additionally, weight gain during early infancy period seems to have a great effect on eruption timing, whereas feeding method's impact is not clarified yet.

Conclusion: Eruption timing of the first primary tooth is not influenced by only one specific factor. Several conditions may act together and increase the risk of delayed or accelerated teeth eruption, occurring additively or even synergistically. The association between perinatal and early infancy factors and eruption timing of the first primary tooth needs further controlled studies as well as research in a wider spread of ethnicities. 
smoking", "gestational age and primary dentition", "premature infant dentition", "birth weight and primary teeth", "breastfeeding and tooth eruption". An additional manual search of the bibliographies of all selected full-text articles and relevant textbooks was performed by the authors.

\section{RESULTS}

\section{A. Maternal Factors}

\section{Smoking during pregnancy / Exposure to smoke}

It was observed that maternal smoking during pregnancy significantly accelerated the onset of primary tooth emergence and a greater progression of eruption over the first years of post-natal life in children whose mothers smoked during pregnancy than those born to non-smokers [7], [10][12].

Three studies stated that no statistically significant differences were observed in eruption time of the first primary tooth as related to smoking during pregnancy, indicating no significant association between early eruption timing of the first primary tooth and the mother's exposure to secondhand smoke during pregnancy [9], [13], [14]. Also, a cross-sectional study in Japan by Hanioka et al. revealed that smoke exposure after birth but no exposure during pregnancy was significantly associated with early tooth eruption [15].

\section{Mother's Vitamin D levels / Sun exposure}

In 2020 Jairam et al. conducted a study to assess the Vitamin D levels and correlate it with the eruption of primary teeth. It was observed that higher the maternal sun exposure during pregnancy, greater the infant's Vitamin D levels soon after birth, preventing infant's Vitamin D deficiency, an etiological factor for delayed eruption of teeth. Vitamin D is important to bone and teeth mineralization because of its role in the maintenance of adequate serum calcium and phosphorus concentrations. Lack of Vitamin D during tooth development may result in enamel and dentin hypoplasia, incomplete development, and delayed tooth eruption [16].

\section{Maternal age}

$\mathrm{Wu}$ et al. conducted a study over a period of 16 months, covering a total of 1296 pregnant woman. The results showed that higher maternal childbearing age was associated with delayed eruption timing of the first primary tooth [9]. In 2016 Lam et al. reported that increasing maternal age was correlated with earlier first tooth eruption [17]. However, Alnemer et al. in 2017, examining 422 women and Sajjadian et al. in 2010, examining 143 women found no relationship between the time of first primary tooth eruption and the maternal age at the time of birth [18], [19].

\section{Maternal diseases}

Aktoren et al. in 2010 found no significant differences in eruption times were recorded in infants whose mothers experienced diseases in the first trimester versus infants whose mothers did not [14].

\section{B. Perinatal Factors}

\section{Gestational Age}

Many studies investigated the possible effect of prematurity on primary tooth eruption. Seow et al. in 1997 concluded that preterm infants have the lowest rates of dental development [21]. It was observed by Aktoren et al. in 2010 that late eruption was seen 3.67 times more in infants who were born at less than 37 weeks [14]. Also, in 2015 Ntani et al. and in 2019 Wang et al. reported that delayed tooth eruption was significantly associated with shorter length of gestation, as well as a negative correlation between the number of erupted teeth in preterm compared to full term infants at the early age ranges [7], [20].

However, four studies reported significant differences between preterm and full-term infants regarding teething time, but this difference was cancelled when a correction with respect to gestational age was made. According to the chronological age, preterm infants showed a delay in the eruption time of the first primary tooth when compared to full-term infants, however when corrected age is considered, no statistically significant differences were found between groups, as premature infants got their first tooth at the same time as full-term infants [22]-[25].

Nevertheless, Chan et al. in 2013 and Yilmaz et al. in 2015 reported no significant difference in teething time between preterm and post-term infants, indicating no association between prematurity and eruption timing of the first deciduous tooth [26], [27].

\section{Birth Weight}

Several studies compared the eruption timing of the first primary tooth in infants in relation to their birth weight. Low birth weight is defined by the World Health Organization as weight at birth less than $2500 \mathrm{~g}$.

According to Delgado et al. in 1975 a significant influence of birth weight on eruption timing of primary teeth was found, as infants with birthweight more than $3 \mathrm{~kg}$ have on average, more erupted teeth than infants with birthweight less than $3 \mathrm{~kg}$ [28]. Neto et al. and Aktoren et al. noted a significant relationship between infant's birth weight and primary tooth emergence, as infants with a birth weight lower than $2500 \mathrm{~g}$ demonstrated significant delayed eruption of the first primary tooth [1], [14].

In 1993 Harris et al. conducted a study, including only infants weighing less than $2000 \mathrm{~g}$, revealing that the earlyforming teeth (incisors, first molars) delayed significantly [29].

Additionally, many studies indicated that very low birthweight $(<1500 \mathrm{~g})$ and especially extremely low birthweight $(<1000 \mathrm{~g})$ is a great risk factor for significant delayed eruption timing of the first primary tooth [23], [24], [26], [31], [32].

Several studies report that birth weight is inversely related to eruption timing of the first primary tooth, suggesting that delayed eruption of the primary teeth may be related to lower birth weight [10], [20], [25], [30]. Also, Ntani et al. and Sajjadian et al. found that primary teeth erupt earlier in infants with higher birth weights [7], [19].

In $2019 \mathrm{Wu}$ et al. found that low birth weight was associated with delayed eruption timing of the first primary tooth, while macrosomia was associated with earlier eruption timing [9]. On the other hand, Garmash et al. reported a slow deciduous teeth growth rate in children born with macrosomia and an increased frequency of deviations in the timing of teeth eruption compared to regional norms [33].

In contrast to the above Alnemer et al. found no relationship between infant's birth weight and the eruption timing of the first primary tooth [18]. 


\section{Birth length}

Four studies have examined the relationship between the length at birth and the emergence of the primary dentition. Two of them found a significant influence of birth length on deciduous teeth eruption, with longer infants having a greater number of primary teeth at one year of age [7], [13]. However, the other two studies indicated no relationship between the birth height of the infant and the eruption timing of primary teeth [18], [19].

\section{Infant sex}

Over the years, many attempts have been made in order to establish the effect of the infant's sex on the eruption timing of the first deciduous tooth. Most of the studies detected no significant difference in the mean eruption times between the genders [1], [4], [14], [19], [23], [25], [30], [34]-[40], such as the study by Al-Batayneh et al in Jordan, covering a total of 1988 children [4]. The same result was noted by Folayan et al. in a cross-sectional study, enrolling 1657 Nigerian children [42]. In complete agreement with those findings are Pavicin's et al. results [24].

In contrast, Infante et al. in 1973 revealed that the deciduous tooth eruption process began at earlier ages in boys than in girls. However, girls surpassed boys in tooth emergence by age of 15 months and remained advanced through completion of the deciduous dentition. The sex differences in the early and later stages of primary teeth emergence stated as significant [43]. Alnemer et al. in 2017 corroborated that a significant association between the gender of the child and the total number of emerged teeth was noted, with girls having more erupted than boys [18]. However, Wu et al. conducted a study in 2019 , mentioning that deciduous teeth erupt earlier in males than in females, as well as Yilmaz et al. in 2017 and Tanguay et al. in 1984 [9], [27], [44].

\section{Infant ethnicity}

The possibility of different eruption timing of the primary teeth between children of different geographic regions has received much attention over the past years. In 2016 Lam et al. elucidated the importance of ethnicity as a determinant of eruption timing of the primary teeth [17]. The same year Warren et al. compared timing of tooth emergence among groups of American Indian, Black and White children at 12 months of age. It was found that tooth emergence occurs at a younger age for American Indian children than it does for White or Black children and no significant differences were detected between Black and White children [36]. Also, Magnusson et al. in 1982 indicated that eruption timing of the first primary tooth is ethnicity and community dependent [37]. Recently, in $2020 \mathrm{Wu}$ et al. confirmed that the eruption timing of the first primary tooth differs depending on infants' ethnicity and community, likely in response to variation in environmental and genetic factors [9].

\section{Early Infancy Period Factors}

\section{Method of Feeding}

Several studies were conducted to assess the possible impact of breastfeeding on the timing of eruption of the primary dentition. A positive correlation was found by Folayan et al. in 2010, noting that primary teeth erupted earlier in children who were exclusively breastfed when compared to those who were partially breastfed [45]. Moreover, Alnemer et al. in 2017 observed that children who were exclusively breast fed were more likely to have an erupted primary tooth, but it was also found that this observation was only significant up to the 8th month of life, following which there was no significant impact of breast feeding on the number of erupted teeth [18]. Holman et al. addressed that partial breastfeeding had no effect on tooth emergence timing, but children who were not breastfed at all showed delayed emergence of the upper incisors [46].

On the other hand, a negative correlation between breastfeeding and eruption timing of the first deciduous tooth was indicated by Zadzinska et al. in 2015, showing that breastfeeding was found to delay the onset of decidious tooth emergence [10]. The same year Ntani et al. also stated that children who were breastfed for longer than seven months had delayed primary tooth eruption and fewer teeth at one and two years. However, this association was only of borderline significance [7].

However, a significant number of studies revealed no effect of breastfeeding on the timing of eruption of the first primary tooth. Chan et al. found no significant differences in age at first primary tooth emergence between exclusively breastfed and exclusively bottle-fed children. In addition, there was no correlation between breastfeeding duration and tooth emergence times [26]. Also, Wu et al. found that neither the breastfeeding model nor the duration of breast feeding were significantly related to the eruption timing of the first primary tooth, as well as Yilmaz et al. in 2015 [9], [27].

\section{Weight gain}

Numerous researchers seek to address the correlation between infant rate of weight gain during the first months of life and the eruption timing of the first primary tooth. Most studies indicate a positive relationship between the weight percentile and number of erupted teeth. Holman et al. in 2005 and Agarwal et al. in 2003 revealed that infants of poor nutritional status exhibit delayed eruption timing of the primary dentition. Eruption may be significantly delayed, even for children of moderate nutritional status, compared to those of good [46], [47]. Also, Verma et al. in 2017 observed that infant's body mass index has direct relationship with the teeth eruption [30].

$\mathrm{Wu}$ et al. proposed that adequate nutrition during early infancy period may prevent delayed eruption timing of the first primary tooth, as well as Lam et al. in 2016, who noted that infant's rate of weight gain during the first 3 months of life was associated with earlier eruption of the first primary tooth [9], [17]. Furthermore, according to Haddad et al. in 2005 , low birth-weight children may have as many teeth as those with a normal birthweight or even more if they catch up with their growth [48].

\section{Infant's Vitamin D level}

In 2020 Jairam et al. conducted a cross sectional study, indicating a strong association between the timing of eruption of primary teeth and Vitamin D deficiency. It was concluded that Vitamin D deficiency could be an etiological factor for delayed eruption [16]. On the contrary in 2015 Yilmaz et al. found no significant relationship between teething time and Vitamin D [27].

\section{Socioeconomic status}

The impact of the socio-economic factor on the dental development of children has been the point of interest for many studies. Enwonwu et al. observed that the average child 
of a high socioeconomic group had 2 to 5 more erupted teeth than a child of a low socio-economic group of comparable age [49]. A significant difference in the number of erupted teeth between children from high socio-economic class when compared with those from low socio-economic class in Nigeria was observed in Oziegbe's et al. study in 2009 [50]. Verma et al. indicated that as status increased a greater number of age-appropriate teeth were present and vice versa [30]. Although, a positive correlation between lower socioeconomic status and higher number of present primary teeth at the age of 2 was found by Ntani et al [7]. In addition, Bambach et al. show no statistically significant social class effect on the number of erupted teeth [34].

\section{DISCUSSION}

During the last decades, many efforts have been made in order to enhance our knowledge about timing and sequence of primary teeth eruption but also about the possible causes that may influence the eruptive process. Genetic contribution has been suggested to be an important determining factor of the tooth emergence timing [8]. In addition, environmental, exogenous factors that could affect the fetus during pregnancy or the infant during the early infancy period seem to have a significant impact on eruption timing of the first deciduous tooth [7], [9], [10], [14]. However numerous investigators have reported contradictory findings.

The effect of maternal factors influencing infant's eruption timing of the first primary tooth has not been fully clarified yet. Maternal smoke exposure during pregnancy is a nonbiological factor affecting the primary dentition; the effect was the reverse of that which might have been expected. Although smoking during pregnancy is associated with preand postnatal growth retardation, most of the studies confirm a positive correlation between maternal smoking and eruption timing of the first deciduous tooth [7], [10], [11], [12], or zero effect of smoking on primary dentition [9], [13]-[15]. The absence of association in some studies could be attributable to different criteria for defining smoking and differing samples sizes followed-up. The potential effect of another maternal factor, childbearing age of the mother on the eruption timing of the primary teeth has not been fully elucidated. Studies have noted positive [17], negative [9] or no significant correlation [18], [19]. The reasons underlying these differences are unclear but may include ethnic or population differences, environmental and genetic factors, as these studies have been assessed in different populations and races. Also, maternal vitamin D levels during pregnancy seem to effect eruption timing of the first primary tooth. Maternal vitamin D deficiency during pregnancy is a risk factor for vitamin D deficiency in infants, an etiological factor for delayed teeth eruption [16].

According to the literature perinatal factors such as gestational age, birth weight and length, infant's sex and ethnicity may affect dental development and timing of tooth eruption. As defined by the World Health Organization, a premature birth is one that occurs before 37 weeks of gestation. Many studies found that eruption timing of the first primary tooth may be delayed in preterm infants. This process may be disrupted because of nutritional deficiencies, exposure to certain medications, duration of oral intubation and traumatic oral manipulations due to prematurity [7], [14], [20], [21]. However, four studies reported that when both chronological age and corrected age were considered, there was no delay in tooth eruption [22]-[25]. Considering that only a few studies in the literature consider the influence of each subcategory of prematurity on eruption timing of primary teeth [22], [26], further studies should be carried out in order to evaluate the effect of severity of prematurity on dental development. A remarkable association also exists between birth weight and eruption timing of the first primary teeth. Low birth weight is defined as a weight at birth of less than 2500 g. Our study revealed that most authors indicated that low birth weight infants are more likely to have delayed primary teeth eruption. On the other hand, birth length does not seem to be a determinant factor. Many studies examined infant's sex as a perinatal factor, affecting teeth eruption timing. Girls are generally more advanced in somatic growth and development than boys up to the preadolescent years. However, some studies have indicated that the eruption process could be independent of the growth process and that boys could show earlier tooth emergence compared to girls [9], [27], [44]. Nonetheless our findings indicated that most of the studies found no association between infants' sex and eruption timing of the first primary tooth [1], [4], [7], [14], [19], [23], [25], [30], [34]-[41]. Additionally, the literature suggests that infant's ethnicity is a determinant factor influencing tooth formation. Eruption timing variations observed within ethnic groups, prove that eruption timing of the first primary tooth may potentially be ethnicity dependent [9], [17], [36], [37]. Further research in a wider spread of races seems necessary in order to obtain a broader view about the impact of ethnicities.

During the early infancy period some postnatal factors could affect the eruption timing of the first primary tooth, as stated in the literature. Feeding method seems to influence the dental occlusion development and the eruption timing of the first primary tooth [7], [10], [18], [45], [46]. Nutritional deficiencies during early postnatal life may cause the development of defective or delayed dentition. Breastfeeding and bottle feeding involve different mechanisms and orofacial muscle activity, which could be one possible reason for the different growth patterns of jaws, dental arch development and tooth eruption. It has been hypothesized that early sucking activity influences the growth of the craniofacial complex and that breast feeding is the ideal stimulus for the physiological development of both the muscular and skeletal components of the orofacial complex [18]. However more research is needed into the possible impact of breast feeding on the emergence of the first primary tooth. Despite the feeding method a significant determinant of primary tooth eruption was proven to be infants' rate of weight gain. Some authors claim that the eruption timing of primary teeth seems to be more affected by postnatal weight gain than by birth weight [9], [17], [30], [46]-[48]. A new field of research is the possible correlation of infant's Vitamin $\mathrm{D}$ deficiency and delayed tooth eruption. The number of studies in the literature examining the effect of Vitamin D on dental development is not adequate, thus highlighting the need for further research. Another exogenous factor, possibly affecting primary teeth eruption chronology, is the socioeconomic status. In our literature review, we found no 
consistency among studies results, maybe due to heterogeneity of the ethnicities examined.

\section{CONCLUSION}

Our study has led us to conclude that according to the literature a strong association exists between birth weight and eruption timing of the first primary tooth, as infants with a low birth weight demonstrating significant delayed eruption. Also, weight gain ratio during the early infancy period seems to affect greatly the eruption chronology, as well as ethnicity. However, eruption timing of the first primary tooth is not influenced by only one specific factor. Several conditions may act together and increase the risk of delayed or accelerated teeth eruption, occurring additively or even synergistically. The association between maternal, perinatal, and early infancy factors and eruption timing of the first primary tooth needs further controlled studies as well as research. Limited studies describing the effect of various neonatal factors on the timing of primary tooth eruption have been reported. More research needs to be done in a wider spread of ethnicities in order to obtain a broader view about the possible impact of climate and nationalities.

\section{REFERENCES}

[1] P. F. Neto and M. Falcao, "Eruption chronology of the first deciduous teeth in children born prematurely with birth weight less than 1500g", Revista Paulista de Pediatria, vol. 32, no. 1, pp. 17-23, March 2014.

[2] A. Nanci, Ten Cate's Oral Histology, 8th ed. Elsevier Health Sciences, 2012.

[3] P. Jain and M. Rathee, "Anatomy, Head and Neck, Tooth Eruption", PubMed, 2021. [Online]. Available: https://pubmed.ncbi.nlm.nih.gov/31751068/.

[4] O. Al-Batayneh, A. Shaweesh and E. Alsoreeky, "Timing and sequence of emergence of deciduous teeth in Jordanian children", Archives of Oral Biology, vol. 60, no. 1, pp. 126-133, September 2015.

[5] H. Alshukairi, "Delayed tooth eruption and its pathogenesis in paediatric patient: a review", Journal of Dental Health, Oral Disorders \& Therapy, vol. 10, no. 3, pp. 209-212, May 2019.

[6] B. Shroff, R. Pileggi, K. Morris, R. Orbegoso, T. Wilson and J. Sauk, "Dynamic variations in the expression of type I collagen and its molecular chaperone Hsp47 in cells of the mouse dental follicle during tooth eruption", Archives of Oral Biology, vol. 39, no. 3, pp. 231-243, March 1994.

[7] G. Ntani et al., "Maternal and early life factors of tooth emergence patterns and number of teeth at 1 and 2 years of age", Journal of Developmental Origins of Health and Disease, vol. 6, no. 4, pp. 299307, May 2015.

[8] D. Pillas et al., "Correction: Genome-Wide Association Study Reveals Multiple Loci Associated with Primary Tooth Development during Infancy", PLoS Genetics, vol. 6, no. 3, March 2010.

[9] H. Wu, T. Chen, Q. Ma, X. Xu, K. Xie and Y. Chen, "Associations of maternal, perinatal and postnatal factors with the eruption timing of the first primary tooth", Scientific Reports, vol. 9, no. 1, February 2019.

[10] E. Żądzińska, A. Sitek and I. Rosset, "Relationship between pre-natal factors, the perinatal environment, motor development in the first year of life and the timing of first deciduous tooth emergence", Annals of Human Biology, vol. 43, no. 1, pp. 25-33, June 2015.

[11] P. Rantakallio and H. Mäkinen, "Number of teeth at the age of one year in relation to maternal smoking", Annals of Human Biology, vol. 11, no. 1, pp. 45-52, January-February 1984.

[12] M. Ounsted, V. Moar and A. Scott, "A longitudinal study of tooth emergence and somatic growth in 697 children from birth to three years", Archives of Oral Biology, vol. 32, no. 11, pp. 787-791, 1987.

[13] J. Bastos, M. Peres, K. Peres and A. Barros, "Infant growth, development and tooth emergence patterns: A longitudinal study from birth to 6 years of age", Archives of Oral Biology, vol. 52, no. 6, pp. 598-606, June 2007.
[14] O. Aktoren, E. B. Tuna, Y. Guven, and G. Gokcay, “A study on neonatal factors and eruption time of primary teeth", Community dental health, vol. 27 no. 1, pp. 52-56, March 2010.

[15] T. Hanioka, M. Ojima, K. Tanaka, N. Taniguchi, K. Shimada and T. Watanabe, "Association between secondhand smoke exposure and early eruption of deciduous teeth: a cross-sectional study", Tobacco Induced Diseases, vol. 16, no., February 2018.

[16] L. S. Jairam, S. Konde, N. S. Raj, N. C. Kumar, "Vitamin D deficiency as an etiological factor in delayed eruption of primary teeth: A crosssectional study ", J Indian Soc Pedod Prev Dent, vol. 38, no. 3, pp.211215, July-September 2020.

[17] C. Un Lam et al., "Influence of metabolic-linked early life factors on the eruption timing of the first primary tooth", Clinical Oral Investigations, vol. 20, no. 8, pp. 1871-1879, November 2015

[18] K. A. Alnemer, S. C. Pani, A. M. Althubaiti, and M. Bawazeer, "Impact of birth characteristics, breast feeding and vital statistics on the eruption of primary teeth among healthy infants in Saudi Arabia: an observational study," BMJ Open, vol. 7, no. 12, p. e018621, 2017.

[19] N. Sajjadian, H. Shajari, R. Jahadi, M. G. Barkett, and A. Sajjadian, "Relationship between birth weight and time of first deciduous tooth eruption in 143 consecutively born infants," Pediatr. Neonatol., vol. 51, no. 4, pp. 235-237, August 2010.

[20] X. Z. Wang et al., "Effects of premature delivery and birth weight on eruption pattern of primary dentition among Beijing children," Chin. J. Dent. Res., vol. 22, no. 2, pp. 131-137, January 2019.

[21] W. K. Seow, "Effect of preterm birth on oral growth and development," Aust. Dent. J., vol. 42, no. 2, pp. 85-91, April 1997.

[22] N. L. Golden, "Teething age in prematurely born infants," Arch Pediatr. Adolesc. Med., vol. 135, no. 10, p. 903, October 1981.

[23] S. R. P. Ramos, R. C. Gugisch, and F. C. Fraiz, "The influence of gestational age and birth weight of the newborn on tooth eruption," $J$. Appl. Oral Sci., vol. 14, no. 4, pp. 228-232, August 2006.

[24] I. S. Pavičin, J. Dumančić, T. Badel, and M. Vodanović, "Timing of emergence of the first primary tooth in preterm and full-term infants," Ann. Anat., vol. 203, pp. 19-23, January 2016.

[25] A. M. Khalifa, R. A. El Gendy, M. M. A. El-Mohsen, A. A. Hammour, and R. S. A. El Lateef Aly, "Relationship between gestational age, birth weight and deciduous tooth eruption," Gaz. Egypt. Paediatr. Assoc., vol. 62 , no. 2, pp. 41-45, June 2014

[26] E. Chan, M. Bockmann, T. Hughes, S. Mihailidis, and G. Townsend, "Do feeding practices, gestation length, and birth weight affect the timing of emergence of the first primary tooth?", in New Directions in Dental Anthropology, G. Townsend, E. Kanazawa, and H. Takayama, Eds. Adelaide: University of Adelaide Press, 2013, pp. 35-45.

[27] A. E. Yilmaz et al., "Teething and affecting factors: experiences from Turkey," Minerva Pediatr., vol. 68, no. 6, pp. 427-434, December 2016.

[28] H. Delgado et al., "Nutritional status and the timing of deciduous tooth eruption," Am. J. Clin. Nutr., vol. 28, no. 3, pp. 216-224, April 1975.

[29] E. F. Harris, B. D. Barcroft, S. Haydar, and B. Haydar, "Delayed tooth formation in low birthweight African-American children," Pediatr. Dent., vol. 15, no. 1, pp. 30-35, January-February 1993.

[30] N. Verma, A. Bansal, P. Tyagi, A. Jain, U. Tiwari, and R. Gupta, "Eruption Chronology in children: A cross-sectional study," Int. J. Clin. Pediatr. Dent., vol. 10, no. 3, pp. 278-282, July-September 2017.

[31] R. M. Viscardi, E. Romberg, and R. G. Abrams, "Delayed primary tooth eruption in premature infants: relationship to neonatal factors," Pediatr. Dent., vol. 16, no. 1, pp. 23-28, January-February 1994.

[32] V. Merglova, L. Hauer, Z. Broukal, J. Dort, and R. Koberova Ivancakova, "General and oral health status of preterm one-year-old very low and extremely low birthweight infants (a cross - sectional study)," Biomed. Pap. Med. Fac. Univ. Palacky Olomouc Czech. Repub., March 2020.

[33] O. Garmash, "Dependence of deciduous tooth eruption terms and tooth growth rate on the weight-height index at birth in macrosomic children over the first year of life," Acta Medica (Hradec Kralove), vol. 62, no. 2, pp. 62-68, January 2019.

[34] M. Bambach, R. Saracci, and H. B. Young, "Emergence of deciduous teeth in Tunisian children in relation to sex and social class," Hum. Biol., vol. 45, no. 3, pp. 435-444, September 1973.

[35] N. L. Soliman, M. A. El-Zainy, R. M. Hassan, and R. M. Aly, "Timing of deciduous teeth emergence in Egyptian children," East. Mediterr. Health J., vol. 17, no. 11, pp. 875-881, November 2011.

[36] J. J. Warren et al., "Timing of primary tooth emergence among U.S. racial and ethnic groups," J. Public Health Dent., vol. 76, no. 4, pp. 259-262, September 2016.

[37] T. E. Magnússon, "Emergence of primary teeth and onset of dental stages in Icelandic children," Community Dent. Oral Epidemiol., vol. 10, no. 2, pp. 91-97, April 1982. 
[38] I. A. McGregor, A. M. Thomson, and W. Z. Billewicz, "The development of primary teeth in children from a group of Gambian villages, and critical examination of its use for estimating age," $\mathrm{Br}$. $J$. Nutr., vol. 22, no. 2, pp. 307-314, May 1968.

[39] E. O. Oziegbe, C. A. Adekoya-Sofowora, T. A. Esan, F. J. Owotade, and M. O. Folayan, "Breastfeeding pattern and eruption of primary teeth in Nigerian children," Pediatr. Dent. J., vol. 20, no. 1, pp. 1-6, January 2010.

[40] J. S. Friedlaender and H. L. Bailit, "Eruption times of the deciduous and permanent teeth of natives on Bougainville Island, Territory of New Guinea: a study of racial variation," Hum. Biol., vol. 41, no. 1, pp. 51-65, February 1969.

[41] A. F. Roche, D. H. Barkla, and J. S. Maritz, "Deciduous eruption in Melbourne children," Aust. Dent. J., vol. 9, no. 2, pp. 106-108, April 1964.

[42] M. Folayan, F. Owotade, E. Adejuyigbe, S. Sen, B. Lawal, and K. Ndukwe, "The timing of eruption of the primary dentition in Nigerian children," Am. J. Phys. Anthropol., vol. 134, no. 4, pp. 443-448, December 2007.

[43] P. F. Infante, "Sex differences in the chronology of deciduous tooth emergence in white and black children," J. Dent. Res., vol. 53, no. 2 , pp. 418-421, March-April 1974.

[44] R. Tanguay, A. Demirjian, and H. W. Thibault, "Sexual dimorphism in the emergence of the deciduous teeth," J. Dent. Res., vol. 63, no. 1, pp. 65-68, January 1984.
[45] M. O. Folayan, E. O. Oziegbe, and A. O. Esan, "Breastfeeding, timing and number of erupted teeth in first twelve months of life in Nigerian children," Eur. Arch. Paediatr. Dent., vol. 11, no. 6, pp. 279-282, December 2010.

[46] D. J. Holman and K. Yamaguchi, "Longitudinal analysis of deciduous tooth emergence: IV. Covariate effects in Japanese children," Am. J. Phys. Anthropol., vol. 126, no. 3, pp. 352-358, March 2005.

[47] K. N. Agarwal, S. Narula, M. M. A. Faridi, and N. Kalra, "Deciduous dentition and enamel defects," Indian Pediatr., vol. 40, no. 2, pp. 124 129, February 2003.

[48] A. E. Haddad and M. S. N. P. Correa, "The relationship between the number of erupted primary teeth and the child's height and weight: a cross-sectional study," J. Clin. Pediatr. Dent., vol. 29, no. 4, pp. $357-$ 362, February 2005.

[49] C. O. Enwonwu, "Influence of socio-economic conditions on dental development in Nigerian children," Arch. Oral Biol., vol. 18, no. 1, pp. 95-107, January 1973.

[50] E. O. Oziegbe, C. Adekoya-Sofowora, M. O. Folayan, T. A. Esan, and F. J. Owotade, "Relationship between socio-demographic and anthropometric variables and number of erupted primary teeth in suburban Nigerian children," Matern. Child Nutr., vol. 5, no. 1, pp. 8692, January 2009. 